\title{
An establishment of the procedures to eliminate incorrect blood due to non-matched or mislabeled tubes
}

\author{
He Ziyi , Wang Qing, Chen Shaobin, Yu Lin, Chen Qingkai \\ Department of Transfusion Research, Dongguan Blood Center, Dongguan 523930, Guangdong, China
}

\begin{abstract}
This study aims to establish a set of procedures to eliminate incorrect blood due to non-matched tubes or mislabeled tubes. The errors such as these are suspected to have occurred when upon first and second-detection. Under the identical laboratory conditions, the results of re-detection of blood bag samples and rare type antigen samples, as well as re-collected samples from donor, and plasma diluted are not consistent with the original results. Here, 20 antigen erythrocytes were detected for blood bag samples and original samples, in which no incorrect blood was mistakenly administered or mislabeled. Samples taken under identical laboratory conditions were not found to have incorrect blood administered to a non-matched tube. The results showed nonlinear changes by HBsAg ELSIA after plasma dilution. The study suggests that the second-detection taken shortly after first detection is the most appropriate method to detect errors at the earliest time point. Blood bag samples are identical to those of the original antigen identification group, which means that the probability of the samples coming from the same donor is extremely highly. At same time, space and plasma diluted, re-detection can effectively exclude incorrect blood being added to a non-matched tube.
\end{abstract}

Keywords: blood type; tube; label; detection; ELISA; dilution

\section{INTRODUCTION}

Blood detection is a necessity for all blood centers in order to check whether there are pathogens residing in the blood, or for blood compatibility examination before a transfusion. However, improper collection, transportation and handling of samples in laboratories can lead to unfortunate results ${ }^{[1]}$. Pre-analysis errors caused by abnormal samples are believed to account for $60 \%-80 \%$ of total errors in laboratories ${ }^{[2]}$. Therefore, it is important to analyze the reasons of erroneous samples and make improvements ${ }^{[3-4]}$. For every blood collection laboratory, the routine management of samples of great importance. For instance, how to avoid errors in the labeling of samples, and ensure the quality of samples. One of the acknowledged methods is the PDCA(plan-do-check-act) cycle management method $^{[5]}$. When samples are collected by blood centers, especially in collective blood donations or blood donation activities with numerous participants, the occurrence of incorrect blood identification becomes a significant issue. When a blood sample from a donor was first tested HBsAg positive, an inconsistency that the blood bag sample was labeled non-reactive was found later. To ensure patient safety, it is extremely important to avoid the inappropriate handling of blood samples and make potential improvements.

In the study, 149 donors' blood samples were involved and the aim is to establish a set of procedures

*Correspondence to: He Ziyi, MD, Department of Transfusion Research, Dongguan Blood Center, 19 Ningjiang Road, Humen Town, Dongguan 523006, Guangdong, China. TEL: +86-769-85152673; E-mail: zyhe_8@163.com.

The authors declared no conflict of interests. 
to eliminate incorrect blood being administered to non-matched or mislabeled tubes.

\section{MATERIALS AND METHODS}

\section{Source of samples}

A total of 149 samples were collected from blood donors at the donation department on Nov. 19, 2018. Three anticoagulant vacuum sample tubes were collected from each donor: 1 tube of EDTA- $\mathrm{K}_{2}$ was used for ELISA and electrochemiluminescence (ECL) detection; The other 2 tubes of EDTA- $\mathrm{K}_{2}$ containing inert separation gel were used for nucleic acid test (NAT) and sample retention, respectively. The special sample transport box was produced by Shenzhen ChunDe Technology Co., LTD.

\section{Reagents and instruments}

ELISA reagents: $\mathrm{HBsAg}$, anti-HCV, HIV-Ag/ $\mathrm{Ab}$ and anti-TP reagents were provided by LiZou and VanTai Biotechnology Co., Ltd., all of which were approved and used within the validity period. Roche CobasTaq Screen MPX version 2.0 (MPX v2.0), Roche Cobas s201 (Roche Diagnostics, Switzerland), Micro lab STAR IVD (Hamilton, Switzerland), Sorvall ST 40R Centrifuge (Thermo Fisher Scientific, USA), HBV DNA standard serum (lot NO. 201709001, concentration: 50U/mL), HCV RNA standard serum (lot NO. 201709001, concentration: 200U/mL), HIV-1 RNA (lot NO. 201709001, concentration: $1000 \mathrm{U} / \mathrm{mL}$ ) for viruses by NAT, were provided by Beijing Control \& Standards Biotechnology Co., Ltd. All reagents were used within the validity period. All instruments were verified or calibrated and were used properly following the protocols. All sample detection operation procedures were performed in accordance with the professional standards set out by our blood center.

\section{Sample test}

Samples were first tested by $\mathrm{HBsAg}$, anti-HCV and HIV Ag/Abby ELISA using two different manufacturers' equipment. All the samples in grey area or reactivity in the first ELISA test were re-tested by double-hole ELISA test. If the double-hole retest was $\mathrm{S} / \mathrm{CO}<0.9$, they were deemed negative; and any of the double-hole retests were $\mathrm{S} / \mathrm{CO} \geqslant 0.9$, they were deemed positive.

\section{Blood typing}

Cell agglutination was used to identify common blood type antigens A, B, D,M,N, S, s, JKa,JKb,Lea, Leb,E, e, C, c, Fya,Fyb,K, Dia,Pl.

\section{Plasma dilution detection}

After dilution the samples were analyzed according to the ELISA process: the chemiluminescence method(ShenZhen YaHuiLong equipment and reagents) was used for detecting $\mathrm{HBsAg}, \mathrm{HBsAb}$, HBeAg, HBeAb, HBcAb, HBsAg, HBsAb, HBeAg, $\mathrm{HBe} A \mathrm{~b}, \mathrm{HBcA}$ band IgM-HBcAb were detected by ELISA using LiZou reagents. Finally, the Rheumatoid Factor was determined. All the above was carried out at Dongguan City's Fifth People's Hospital.

\section{Nucleic acid test(NAT)}

The Roche Cobas s201 NAT system first tested mixed samples of 6 samples. If the mixed samples were reactive, the single-sample test was performed. In this way, HBV/HIV/HCV viruses can be detected simultaneously.

\section{RESULTS}

\section{Results of HBsAg-ELISA and NAT}

Results from the first and the second tests containing two HBsAg-ELISA reagents; and NAT results on the second and the third tests(Table 1).

\section{Results of re-detection on half an hour before and after blood donation}

A total of 19 blood bag samples were tested by ELISA double-hole test for 30 minutes before and after blood donation. The results were all insignificant.

\section{Results of re-detection of the same space-time}

149 blood bag samples collected by the No. 2 Blood Collection Vehicle on the same day were re-tested by

Table 1 Results of three times HBsAg-ELISA and NAT

\begin{tabular}{|c|c|c|c|}
\hline \multirow{2}{*}{ Reagent } & \multicolumn{2}{|c|}{ ELISA for the first and secondary detection } & \multirow{2}{*}{$\begin{array}{c}\text { The third ELISA } \\
\text { Results(s/co) }\end{array}$} \\
\hline & First results(s/co) & Second results(s/co) & \\
\hline \multirow[t]{4}{*}{ HBsAg-VanTai (s/co) } & Posi tive $(28.57)^{*}$ & Positive $(26.90)^{*}$ & Positive(28.57) \\
\hline & & Negative $(0.10)^{* * *}$ & Negative $(0.11)^{* *}$ \\
\hline & & & Positive $(28.57)^{\#}$ \\
\hline & & & Positive $(21.74)^{\# \#}$ \\
\hline \multirow[t]{2}{*}{ HBsAg-LiZon(s/co) } & Negative $(0.14)^{*}$ & Negative $(0.10)^{*}$ & Negative $(0.06)^{\#}$ \\
\hline & & Negative $(0.06)^{* *}$ & Negative $(0.10)^{\# \#}$ \\
\hline $\operatorname{Roche}(\mathrm{Ct})$ & l & Negative & Negative \\
\hline
\end{tabular}


single-hole ELISA. The results were all insignificant.

\section{Results of 20 blood group antigens}

The donors' original sample and blood bag sam- ple were tested for consistencies in antigens. A total of 20 blood group antigens were tested, and the results showed that the response intensity was consistent(Table 2).

Table 2 Agglutination intensity of 20 blood type antigens on the original sample and the blood bag sample

\begin{tabular}{ccccccccccccc}
\hline Group antigen & A & B & D & M & N & S & s & $\mathrm{Jk}^{\mathrm{a}}$ & $\mathrm{Jk}^{\mathrm{b}}$ & $\mathrm{Le}^{\mathrm{a}}$ \\
\hline Original sample & $4+$ & $4+$ & $4+$ & $4+$ & - & - & $4+$ & $4+$ & $4+$ & - \\
Blood bag sample & $4+$ & $4+$ & $4+$ & $4+$ & - & - & $4+$ & $4+$ & $4+$ & - \\
Group antigen & $\mathrm{E}$ & $\mathrm{e}$ & $\mathrm{c}$ & $\mathrm{C}$ & $\mathrm{Fy}^{\mathrm{a}}$ & $\mathrm{Fyb}$ & $\mathrm{K}$ & $\mathrm{Di}^{\mathrm{a}}$ & $\mathrm{Pl}$ & $\mathrm{Le}^{\mathrm{b}}$ \\
Original sample & $4+$ & $4+$ & $4+$ & $4+$ & $1+$ & - & - & - & - & $1+$ \\
Blood bag sample & $4+$ & $4+$ & $4+$ & $4+$ & $1+$ & - & - & - & - & $1+$ \\
\hline
\end{tabular}

Notes:+,agglutination; -,no agglutination.

\section{Results of three recollected donor samples}

This table includes one ELISA (E2) sample, one sample retained for two years (R2), and one sample placed in an erythrocyte preservation solution (including $1 \mathrm{~mL}$ of preservation solution) (P2). The three samples were tested with VanTai and LiZou reagents respectively, and the results are shown in the Table 3.The detection results from two reagents were quite different, which may be because of the different coating substrates.

Table 3 The results of recollected samples and retained samples

\begin{tabular}{lccccrrr}
\hline & Original & Original sample & Two-year retention & Two-year retention & E2 & R2 & P2 \\
& sample & dilution(1:1) & sample dilution (1:4) & sample dilution (1:8) & 7.98 & 28.57 & 22.84 \\
\hline VanTai(s/co) & 28.57 & 28.57 & 18.93 & $/$ & 2.34 \\
LiZou(s/co) & $/$ & 0.08 & $/$ & $/$ & 0.06 & 0.02 & 0.05 \\
\hline
\end{tabular}

Notes: / means undone.

\section{Exclusion test on cross-reactive protein}

The ELISA anti-TP (VanTai reagent) plates were used to detect HBsAg on the above original sample and blood bag sample. After HBsAg-VanTai procedures, the results were insignificant. This means the test can be ruled out for high adhesion and reaction with the HBsAg enzyme marker protein.

\section{Exclusion test of Hook effect}

The original sample plasma and ultra-pure water, and blood bag samples for retesting were diluted in accordance with dilutions: 1:1, 1:2, 1:4 and 1:8 with the original samples treated with VanTai reagent. The samples retained for two years were tested by ELISA, respectively. The plasma and ultra-pure water in the original sample were diluted in accordance with 1:1, 1:2, 1:4 and 1:8 with LiZou reagent, and the results were detected by ELISA, as shown in Table 4 .

\section{Other supplementary tests}

Chemiluminescence assay was used to detect for HBsAg, HBsAb, HBeAg, HBeAb, HBcAb of hepatitis $B$. The results showed that only the HBsAb result was positively significant, while the other data were insignificant. ELISA was used to detect for $\mathrm{HBsAg}$, HBsAb, HBeAg, HBeAb, HBcAb and HBcAb-IgM. The results show that only HBsAb was positive and
Table 4 The results of original samples, blood bag samples, two years retention samples and ultra-pure water ratio dilution by ELISA

\begin{tabular}{|cc}
\hline Samples & VanTai(s/co) \\
\hline Ultra-pure water & 0.02 \\
\hline Original sample & \\
\hline 1:1 Dilution & 28.57 \\
\hline 1:2 Dilution & 28.57 \\
\hline 1:4 Dilution & 20.01 \\
\hline 1:8 Dilution & 2.40 \\
\hline Blood bag sample & 0.01 \\
\hline 1:1 Dilution & 2.50 \\
\hline 1:2 Dilution & 3.90 \\
\hline 1:4 Dilution & 2.75 \\
\hline 1:8 Dilution & 0.20 \\
\hline Two-year retention sample & 1.72 \\
\hline 1:1 Dilution & 6.74 \\
\hline 1:2 Dilution & 5.03 \\
\hline 1:4 Dilution & 1.90 \\
\hline 1:8 Dilution & 1.90 \\
\hline
\end{tabular}

the others were negative. The results of the rheumatoid factor test were normal.

\section{DISCUSSION}

Although technology continues to evolve, human errors in the blood storage process are undesirable, such as incorrect sample collecting, transporting and testing, as they can directly affect blood safety and detection quality ${ }^{[6,7]}$. The implementation of whole rocess quality control measures can effectively reduce 
the error rate in testing, eliminate the influence of adverse factors, and improve the accuracy of results ${ }^{[8]}$. In May 2006, the Ministry of Health of Chinaissued the "Standards for the Quality Management of Blood Center Laboratories" edict, which set requirements for the management of key control points before, during and after the testing of samples, to ensure standards of accuracy and effectiveness ${ }^{[9]}$. A previous study reported that the implementation of work standardization, institutionalized management and standardized operation measures can effectively avoid negative impacts ${ }^{[10]}$. Sample collection is a nursing operation, which often is the weakest link in the quality management of medical laboratories, due to the lack of quality control in nursing technology and related procedures ${ }^{[11]}$. The incidence rate of unqualified specimens reported in China in 2008 reached $9.4 \%$, of which $80 \%$ of all unsatisfactory test results could be traced to the unqualified quality of samples ${ }^{[1]}$.

Generally, for the same sample, even if different ELISA reagents are used, the difference in s/co value will not be significant. When the first reagent is detected as negative or positive, the same attests for the second reagent. So, if the first reagent detects a negative result and the second reagent shows strong positive (for e.g. the s/co a first result was $0-0.2$ for the negative, and second 10.0-28.0 for the positive), with a difference of ten to hundreds of times, this error exceeds the inter-group error between reagent tests $(20 \%)$. Therefore, the generation of such large errors should be considered as coming from different samples, with possible reasons such as 1)confused blood samples, and / or 2)incorrect blood collection labeling or tube. Possible consequences may be that clear samples (without HBV/HIV/HCV/TP) might be discarded as positive blood after detection and blood with HBV/ HIV/HCV/TP might have the possibility of being distributed to patients as negative, leading to serious occurrences of blood transmitted diseases.

This study illustrated that when the results of the original sample and the blood bag sample shows at first negative, and second as a positive, great attention should be paid as to how the error occurred. Even if there is no negative or positive reaction, certainly if the difference in the degree of reaction exceeds $20 \%$, blood collection and administering staff should be highly alerted. Although this is of no practical significance, it may serve as a warning as to other possible errors in the blood collection department, and help to improve the monitoring of the clinic's procedures. Precautions, such as the same blood collection department for all procedures, or all blood and blood bag sample re-testing administered sequentially (one after another at a short time interval) can be considered as effective ways of detecting errors. In this case, the results of the 149 retested blood bag samples and matching blood bags were not found to be incorrect. The second method is to identify the blood type of the original sample and the blood bag sample. In the event of even one blood type antigen found inconsistent between the two samples, they can be immediately judged as being two different samples. Thus, 20 blood type antigens from the original sample and matching blood bag sample were identified, with identical antigenic reactivity intensity. So in short, no error was found. The third method is to collect a second sample from the same donor, and then perform an ELISA test. If the results of the two samples are obviously inconsistent, they can be judged as coming from different samples. However, with this method, if the samples were collected and tested again, the possibility of the wrong tube and/or wrong label cannot be confirmed. If the above three methods are unable to find the reason, it may be caused by the reagent or the donated sample. Sometimes when the blood is diluted multiple times for the ELISA test, the hook effect or other immunological abnormalities can occur. The fifth method is to adopt other methods. In this case, chemiluminescence was used to detect $\mathrm{HBsAg}$, HBsAb, $\mathrm{HBeAg}, \mathrm{HBeAb}$, HBcAbfor hepatitis B. If the donor was infected with $\mathrm{HBV}$, the reaction pattern would be different from that of a non-infected person. Here, the results of the original sample, the blood bag sample and the second collected sample were all HBsAb positive, which did not meet the characteristics of positive HBV infection. It can be inferred from the above investigation that the clinic had not made any mistakes, such as administering incorrect blood to the wrong tube, or incorrectly labeling any blood. It was caused by a specially immunological factors.

In conclusion, the erroneous sample may have been due to a VanTai non-specific reaction reagent. It is inferred that the incorrect maintenance of a reagent may lead to a decrease or blockage in its reactivity. However, when the sample was diluted with ultrapure water after normal detection, it was found that a special immunological reaction had occurred, which was the first known occurrence in Dongguan Blood Center.

\section{References}

[1] Li RQ, Gong LJ, Wang TJ, et al. Application of sigma method in clinical biochemical test quality management[J]. Chin J Lab Med, 2017, 40(9):727-732

[2] Hu DM, Zhou YL, Jy L, et al. ISO 15189:2012 interpretation of key changes in technical requirements[J]. Chin 
J Clin Lab Manage, 2014, 2(3):162

[3] Chen XG, Wu H, Sun HJ. Effect of continuous quality improvement on prognosis of maintenance hemodialysis patients[J].B eijing Med, 2011, 33(2):111-115

[4] Yu ZM. Application of continuous quality improvement in urine sample collection of patients with nephropathy[J]. Zhonghua Hu Li Za Zhi (in Chinese), 2010, 45(7):613614

[5] Liu WX, Yc T, Zhu H, et al. Application of PDCA in medical laboratory sample management[J]. Jilin Medical Science (in Chinese), 2013, 33(34):7330-7331

[6] Luan GY. Analysis of quality control management measures in the whole process of blood sample examination[J]. Electro J Clin Med Liter, 2015, 2 (12) : 2271-2272.

[7] Chen ZX. Quality control of clinical biochemical exami- nation of blood samples[J]. Modern Diag Treat, 2014, 25(23):5300-5302

[8] Wu Q, WeiRJ, Zhang J. Analysis of key points of quality control in each Link of blood sample examination[J]. Biotech World, 2015, 11(12):240-242.

[9] Jy S. Application of quality management control in blood sample examination[J]. Med Inform, 2012, 25(12):41412

[10] Wang YG. Research and application of quality control in blood sample test[J]. Chin Foreign Med J, 2012, 31(4) : 26-27.

[11] Rong GR, Zhang PP, Zhao LM, et al. Quality control of blood specimen collection and transportation[J]. Chin J Nurse, 2008, 43(7):645-647

(Received 8 May 2019, Revised 5 August 2019, Accepted 29 August 2019) 